\title{
LETTER TO THE EDITOR Necroptosis of tumor cells leads to tumor necrosis and promotes tumor metastasis
}

Cell Research (2018) 28:868-870; https://doi.org/10.1038/s41422018-0058-y

\section{Dear Editor,}

Foci of cell death are commonly observed in core regions of solid tumors as a result of inadequate vascularization and subsequent metabolic stresses such as hypoxia and glucose deprivation. Since the morphology of dead tumor cells appears to be necrotic, it is often referred as tumor necrosis. Tumor necrosis has been found to be associated with tumorigenesis and poor prognosis for years, ${ }^{1}$ but the role of necrosis in tumor development is still largely unknown. Additionally, the molecular mechanism of necrosis is an under-studied area due to the lack of experimental system for manipulating necrotic cell death. Necroptosis is a form of programmed, caspase-independent necrosis. ${ }^{2}$ Studies on TNF-induced necroptosis have improved our understanding on the molecular mechanism of necroptosis. It is now known that the protein kinases receptor interacting protein kinase 1 (RIPK1), RIPK3 and the mixed lineage kinase domain-like (MLKL) constitute the core of the necroptosis machinery. ${ }^{3-5}$ Importantly, RIPK3-mediated phosphorylation of MLKL results in MLKL oligomerization and its subsequent translocation to the plasma membrane. ${ }^{6}$ Necroptotic pathway may be involved in tumorigenesis, ${ }^{7,8}$ yet it is not clear if tumor cells undergo necroptosis during tumor development and if so, what role does necroptosis play in tumorigenesis and tumor metastasis. In this study, we demonstrated that necroptosis of tumor cells is responsible for tumor necrosis and is critical for metastasis.

Tumor necrosis was often observed in the core regions of solid tumors that had reached a certain size as seen in the MMVT-PyMT breast tumor (Supplementary information, Figure S1a). ${ }^{9}$ Although necroptotic pathway has been reported involved in tumor development, ${ }^{8}$ it is unclear whether tumor cells undergo necroptosis during tumor progression, and whether necroptosis plays any role in tumor necrosis. To explore the potential involvement of necroptosis in tumor necrosis, we first examined the expression of MLKL protein and its phosphorylation status, which is the hallmark of activated necroptosis, ${ }^{3,4}$ in different MMVT-PyMT breast tumor samples by immunohistochemistry. We found that MLKL expression levels were dramatically elevated in tumor cells during tumor growth and the phosphorylation of MLKL was detected specifically in cells around necrotic areas (Fig. 1a and Supplementary information, Figure S1b). This result suggests necroptosis may be engaged in dying cells within necrotic areas, which was confirmed by the presence of oligomerized MLKL, another indication of necroptosis activation, in tumor samples with tumor necrosis (Fig. 1b). Since RIPK3 expression is reduced in tumors due to increased DNA methylation, ${ }^{10}$ we also checked RIPK3 protein level in MMVT-PyMT tumors. As shown in Fig. 1b, middle panel, RIPK3 expression was mild in the early stages of the MMVT-PyMT tumors, but significantly increased in the late stages, which bear tumor necrosis. The engagement of necroptosis in tumor necrosis was also observed in human breast, lung, and liver cancer samples (Fig. 1c, d and Supplementary information, Figure. S1c). These results indicate that necroptosis in tumor necrotic areas is a general phenomenon in solid tumors.

To investigate the role of necroptosis in tumor necrosis and tumorigenesis, we examined whether the loss of MLKL in tumor cells affects tumor necrosis, tumor development and tumor metastasis in an orthotopic breast cancer model. In this model, breast cancer cells, MVT-1, were implanted in the mammary fat pads of mice, where breast tumor developed and then metastasized to the lung within four weeks. ${ }^{11}$ Similar to MMTV-PyMT tumors, MVT-1 breast tumors also showed necrosis and phosphorylated MLKL expression in tumor cells near necrotic areas (Fig. 1e). We generated MLKL KO MVT-1 cells with CRISPR/Cas9 technology (Supplementary information, Figure S2a), and found MLKL deletion did not affect the in vitro proliferation and migration of MVT-1 cells (Supplementary information, Figure S2b and S2c).

WT and MLKL KO MVT-1 tumors grew at a similar rate until 4 weeks after implantation (Fig. If and Supplementary information, Fig. S2d). However, by 5 weeks, MLKL KO tumors were slightly smaller than WT tumors (Fig. 1f). Besides, cell death was dramatically reduced in MLKL KO tumors compared to WT tumors (Fig. $1 \mathrm{~g}$ and Supplementary information, Figure S2e). In this model, WT host cells including immune cells infiltrate into MVT-1 tumors, we thus examined the levels of MLKL and phosphorylated MLKL in WT and MLKL KO tumors. Similar as seen in Fig. 1e, MLKL protein was highly expressed in WT tumor cells and phosphorylated MLKL was detected in cells within necrotic areas (Supplementary information, Figure. S3a). As expected, MLKL expression was detected in infiltrating host cells, but not in MLKL KO tumor cells (Supplementary information, Fig. S3b). However, almost no phosphorylated MLKL was observed in MLKL KO tumors (Supplementary information, Fig. S3b), indicating that the infiltrating host cells are not as sensitive as tumor cells to necroptosis. These results also confirmed our conclusion that necroptosis occurs in tumor cells near necrotic cores. Since abolishing necroptosis of tumor cells did not completely block tumor cell death (Fig. 1g, Supplementary information, Figure S2e and S3b), we then further examined tumor death in MLKL KO tumors. Most cells in the dead areas of WT tumors had typical necrotic morphology - rounded with intact nuclei, while those in the dead areas of MLKL KO tumors were broken down with fragmented nuclei (Fig. 1h), which raised the possibility that tumor cell death may switch to apoptosis when necroptosis is blocked in MLKL KO tumors. To explore this possibility, we performed TUNEL assay, a widely-used method to detect apoptotic cell death in 
a
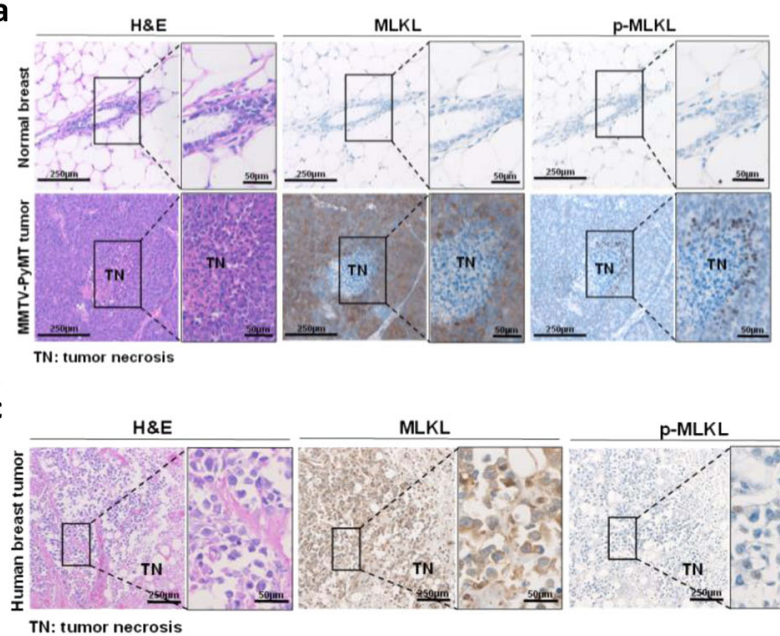

e

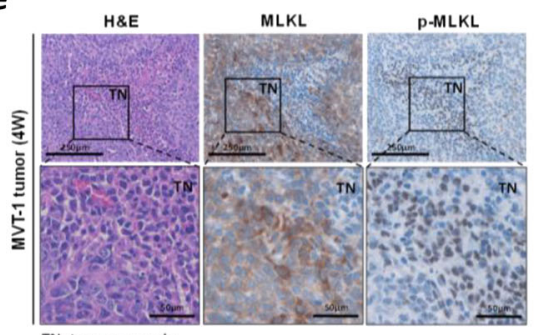

h

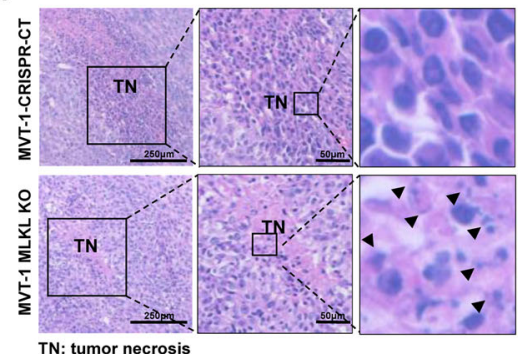

j

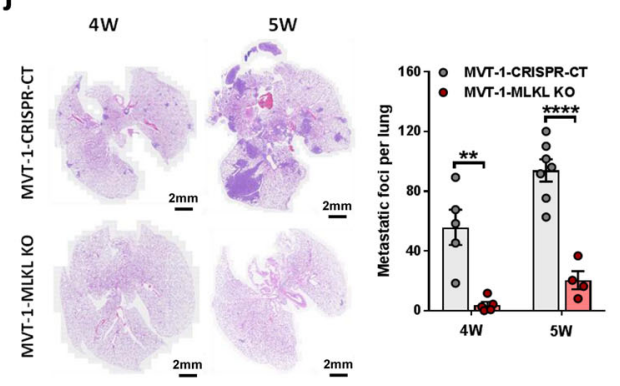

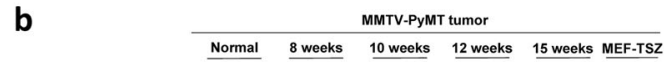

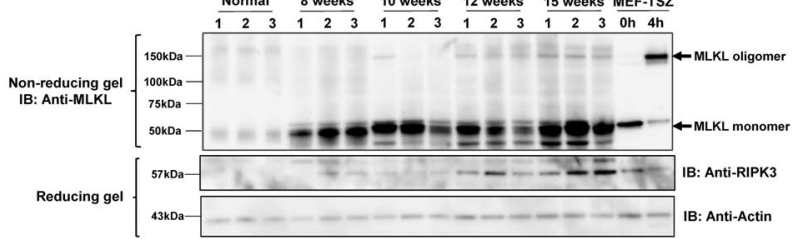

g

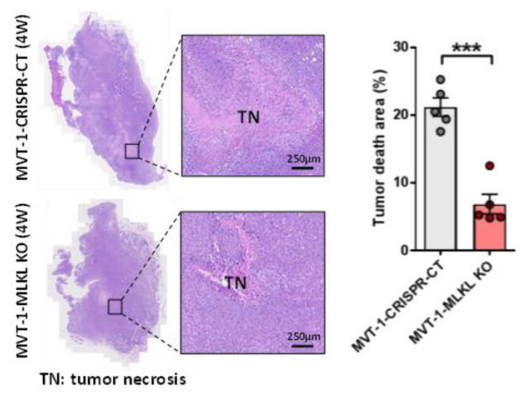

i

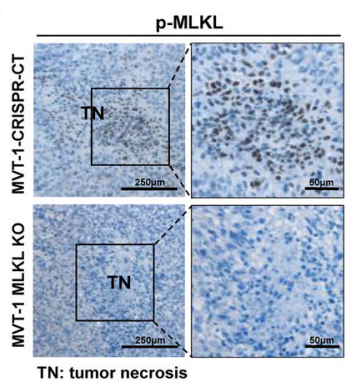

k

MVT-1-CRISPR-CT

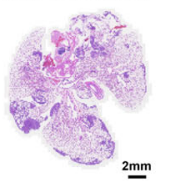

MVT-1-MLKL KO

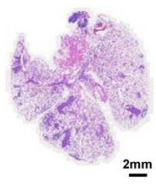

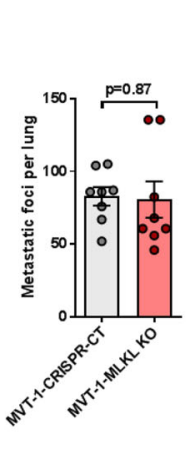

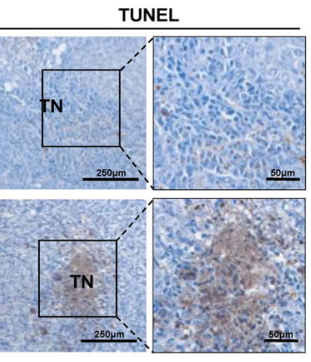

MVT-1-CRISPR-CT MVT-1-MLKL KO Mixing

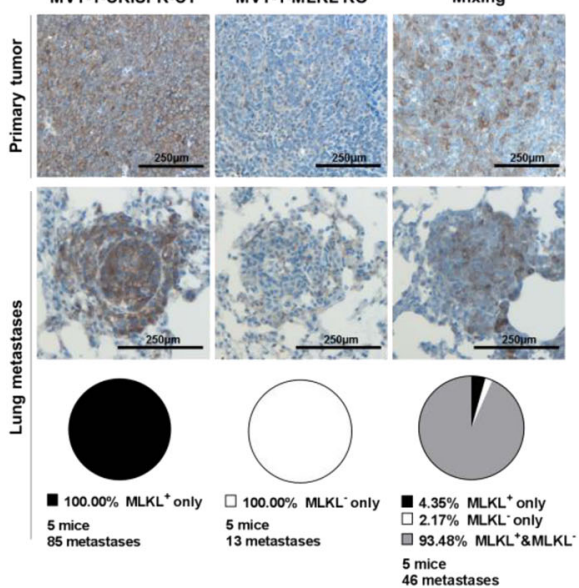

these tumor samples. Much fewer cells were TUNEL positive in the dead area of WT tumors compared to those in MLKL KO tumors (Fig. 1i). These results indicated that necroptosis, but not apoptosis, occurs in the dead areas of WT tumors and blocking necroptosis switches tumor necrosis to tumor apoptosis. Surprisingly, tumor metastasis to the lung was almost completely abolished in MLKL KO MVT-1 cell-implanted mice (Fig. 1j). We also generated MLKL knockdown MVT-1 cells and similar results of tumor necrosis, tumor growth and tumor metastasis were observed (data not shown). As a control, we intravenously injected WT or MLKL KO MVT-1 cells into WT FVB mice through tail vein and found no difference in lung metastasis (Fig. 1k). These 
Fig. 1 Necroptosis in tumor necrosis and metastasis. a Representative image of stained sections of normal or MMTV-PyMT tumor breast tissue. Serial sections were stained with H\&E or immunohistological staining with anti-MLKL or anti-pMLKL antibodies. 100\% live tumor cells are MLKL positive, and $88.17 \pm 12.51 \mathrm{p}$-MLKL positive cells are observed each field $\left(3 \times 10^{5} \mu \mathrm{m}^{2}\right)$. b Western blot analysis of normal breast tissue and MMTV-PyMT tumor tissues. Cell lysates of untreated or $4 \mathrm{~h}$ TSZ treated MEFs serve as control for MLKL monomer and MLKL oligomer. c Representative image of stained sections of human tumor breast tissue. Stained with H\&E or immunohistological staining with anti-MLKL or anti-pMLKL antibodies. $100 \%$ live tumor cells are MLKL positive, and $77.67 \pm 10.48 \mathrm{p}-\mathrm{MLKL}$ positive cells are observed each field $\left(3 \times 10^{5} \mathrm{~mm}^{2}\right)$. d Western blot analysis of human normal and tumor breast tissue. Cell lysates of untreated or $4 \mathrm{~h}$ TSZ treated HT29 cells serve as control for activation of phosphorylated MLKL. e Representative image of stained sections of MVT- 1 tumor tissue. MVT- 1 cells $\left(2 \times 10^{6} / \mathrm{mouse}\right)$ were injected into the right inguinal mammary fat pad of FVB/NJ mice. Tumors were collected at 4 weeks and serial sections were stained with H\&E or immunohistological staining with anti-MLKL or anti-pMLKL antibodies. $100 \%$ live tumor cells are MLKL positive, and $264.8 \pm 29.9$ $\mathrm{p}-\mathrm{MLKL}$ positive cells are observed each field $\left(3 \times 10^{5} \mu \mathrm{m}^{2}\right)$. $\mathbf{f}$ Tumor volume in mice injected with MVT-1-CRISPR-CT or MVT-1-MLKL KO cells was measured weekly. $g$ Representative image of H\&E stained tumors (left panel) at 4weeks from mice injected with MVT-1-CRISPR-CT or MVT1 -MLKL KO cells. Percent of tumor necrotic area (right panel) of total tumor from mice at 4 weeks. ${ }^{* * *} P<0.001$. $\mathbf{h}$ Representative image of H\&E stained 4-week tumors from FVB/NJ mice injected with MVT-1-CRISPR-CT or MVT-1-MLKL KO cells. i Representative image of tumor sections identifying areas of apoptosis by TUNEL staining from 4-week tumors of FVB/NJ mice injected with MVT-1-CRISPR-CT or MVT-1-MLKL KO cells. j Representative image of H\&E stained lung sections (left panel) from mice 4 and 5 weeks after injected with MVT-1-CRISPR-CT or MVT-1-MLKL KO cells showing lung metastasis. Total lung metastasis was determined by counting the number of metastatic foci per lung. ${ }^{* * P}<0.01,{ }^{* * * * P}$ $<0.0001$. k Representative image of lungs collected from mice i.v. injected with $1 \times 10^{6}$ either MVT-1-CRISPR-CT or MV-1 MLKL KO cells for 2 weeks. Number of metastatic foci per lung was counted. I MVT-1-CRISPR-CT cells, MVT-1-MLKL KO cells, or $1: 1 \mathrm{mixed}$ cells $\left(2 \times 10^{6} / \mathrm{mouse}\right)$ were injected into the right inguinal mammary fat pad of FVB/NJ mice. Representative image of MLKL stained primary tumor (upper panel) and lung metastases (lower panel) is shown. Based on MLKL staining, lung metastases were counted and each focus was categorized as either MLKL positive (MLKL ${ }^{+}$only), MLKL negative (MLKL ${ }^{-}$only), or with both $M L K L$ positive and negative staining $\left(\mathrm{MLKL}^{+} \& \mathrm{MLKL}^{-}\right)$

results indicated that the inhibitory effect of MLKL deletion on lung metastasis is not due to altering the migration ability of MVT1 cells in vivo. To further exclude the possibility that MLKL deletion alters the metastasis ability of tumor cells, we injected 1:1 mixed WT and MLKL KO MVT-1 cells and examined the lung metastasis of these cells by detecting MLKL expression in lung foci. As shown in Fig. 1l, upper panel, mixing of WT and MLKL KO cells did not affect the growth of primary breast tumors and both cells grew evenly in these tumors. More importantly, $>90 \%$ of lung metastases in the mixing group showed polyclonal migration of both WT and KO cells (Fig. 1I, lower panel), consistent with previous finding, ${ }^{12}$ indicating that there is no metastasis defect of MLKL KO MVT-1 cells to the lung. Our data suggest MLKL mediated necroptosis in tumor cells leads to tumor necrosis and is critical for lung metastasis. As shown in Supplementary information, Figure S4a, S4b and S4c, production of inflammatory cytokines, IL-1 $\beta$ and TNFa, was significantly reduced in macrophages from MLKL KO tumors, indicating the contribution of necroptosis-induced inflammation to the tumorigenesis difference between WT and MLKL KO tumors.

Although tumor necrosis is often seen in aggressive solid tumors with poor prognosis, the underlying mechanism of tumor necrosis and its role in tumor development and tumor metastasis is largely unknown. In this study, we showed for the first time that tumor cells undergo necroptosis under physio-pathological conditions, in areas of tumor necrosis in murine and human solid cancers. Our study provides the first in vivo evidence for the critical role of tumor cell necroptosis in tumor growth and metastasis, raising the possibility to develop novel cancer treatment strategy by targeting necroptosis.

\section{ACKNOWLEDGEMENTS}

We thank Dr. Glenn Merlino for his critical suggestions on this project. This research was supported by the Intramural Research Program of the Center for Cancer Research, National Cancer Institute, National Institutes of Health. We gratefully acknowledge the assistance of Alex Alfortish and Celeste Faia, and the Louisiana Cancer Research Consortium (LCRC) Biospecimen Core Laboratory for provision of the tissue samples. (http://www.louisianacancercenter.org/research/shared-resources/ biospecimen-core/)

\section{ADDITIONAL INFORMATION}

Supplementary information accompanies this paper at https://doi.org/10.1038/ s41422-018-0058-y.
Competing interests: The authors declare no competing interests.

Delong Jiao ${ }^{1}$, Zhenyu Cai ${ }^{1,2}$, Swati Choksi ${ }^{1}$, Dan Ma ${ }^{1}$, Moran Choe ${ }^{1}$, Hyung-Joon Kwon ${ }^{1}$, Jin Young Baik ${ }^{1}$, Brian G. Rowan ${ }^{3}$, Chengyu Liu ${ }^{4}$ and Zheng-gang Liu ${ }^{1}$ ${ }^{1}$ Center for Cancer Research, National Cancer Institute, National Institutes of Health, 37 Convent Drive, Bethesda, MD, 20892, USA;

${ }^{2}$ National Center for Liver Cancer, Eastern Hepatobiliary Surgery Hospital, Shanghai, China; ${ }^{3}$ School of Medicine, Tulane University, New Orleans, LA 70112, USA and ${ }^{4}$ Transgenic Core, National Heart Lung and Blood Institute; National Institutes of Health, 37 Convent Drive, Bethesda, MD 20892, USA

These authors contributed equally: Delong Jiao, Zhenyu Cai. Correspondence: Zheng-gang Liu (zgliu@helix.nih.gov)

\section{REFERENCES}

1. Richards, C. H., Mohammed, Z., Qayyum, T., Horgan, P. G. \& McMillan, D. C. The prognostic value of histological tumor necrosis in solid organ malignant disease: a systematic review. Future Oncol. 7, 1223-1235 (2011).

2. Galluzzi, L. \& Kroemer, G. Necroptosis: a specialized pathway of programmed necrosis. Cell 135, 1161-1163 (2008).

3. Zhao, J. et al. Mixed lineage kinase domain-like is a key receptor interacting protein 3 downstream component of TNF-induced necrosis. Proc. Natl. Acad. Sci. USA 109, 5322-5327 (2012).

4. Sun, L. et al. Mixed lineage kinase domain-like protein mediates necrosis signaling downstream of RIP3 kinase. Cell 148, 213-227 (2012).

5. Ofengeim, D. \& Yuan, J. Regulation of RIP1 kinase signalling at the crossroads of inflammation and cell death. Nat. Rev. Mol. Cell Biol. 14, 727-736 (2013).

6. Cai, Z. et al. Plasma membrane translocation of trimerized MLKL protein is required for TNF-induced necroptosis. Nat. Cell Biol. 16, 55-65 (2014).

7. Seifert, $L$. et al. The necrosome promotes pancreatic oncogenesis via CXCL1 and Mincle-induced immune suppression. Nature 532, 245-249 (2016).

8. Lalaoui, N. \& Brumatti, G. Relevance of necroptosis in cancer. Immunol. Cell Biol. 95, 137-145 (2017).

9. Lin, E. Y. et al. Progression to malignancy in the polyoma middle T oncoprotein mouse breast cancer model provides a reliable model for human diseases. Am. J. Pathol. 163, 2113-2126 (2003).

10. Koo, G. B. et al. Methylation-dependent loss of RIP3 expression in cancer represses programmed necrosis in response to chemotherapeutics. Cell Res. 25, 707-725 (2015).

11. Trikha, P. et al. E2f3 in tumor macrophages promotes lung metastasis. Oncogene 35, 3636-3646 (2016).

12. Cheung, K. J. et al. Polyclonal breast cancer metastases arise from collective dissemination of keratin 14-expressing tumor cell clusters. Proc. Natl Acad. Sci. USA 113, E854-E863 (2016). 